\title{
Rally Labinoamericano de Innovación: una experiencia para el desarrollo de competencias profesionales
}

(C) Copyright 2021. Universidad Nacional Autónoma de Nicaragua, Managua (UNAN-Managua) Todos los derechos reservados

\section{Latin American Innovation Rally: an experience for the development of professional competences}

\section{Marcos Antonio Reyes Centeno}

Vice Decano de Facultad Regional Multidisciplinaria de Chontales UNAN - Managua

Docente del departamento de Ciencias de la Educación y Humanidades

marco.reyes@unan.edu.ni

https://orcid.org/0000-0003-1023-3724

\section{Karla Patricia Castilla}

Docente del Departamento de Ciencias, Tecnología y Salud

Coordinadora de Extensión Universitaria

UNAN Managua - FAREM Chontales

Karla.castilla@unan.edu.ni

https://orcid.org/0000-0001-7641-067X

Fecha de recibido: 26/04/2021

\section{Resumen}

En este artículo, que nace de la sistematización de una experiencia de extensión, se abordan de manera taxativa aspectos torales sobre el rally latinoamericano de innovación y su incidencia en el desarrollo de competencias genéricas y profesionales en los estudiantes que participan en este evento. El rally latinoamericano de innovación, se desarrolla todos los años en las Facultades de la UNAN-Managua, al igual que en otras universidades de Latinoamérica. El objetivo fundamental del rally, es el desarrollo de competencias para la innovación en los estudiantes que se están formando en las universidades, además

\section{Yesenia Sobeyda Téllez Gómez}

Docente del Departamento de Ciencias, Tecnología y Salud UNAN-Managua, FAREM-Chontales yesenia.tellez@unan.edu.ni https://orcid.org/0000-0002-7493-2615
Fecha de dictaminado: 15/05/2021

de los mentores, docentes y otros profesionales que conducen el proceso de definición y operativización de las ideas de los jóvenes.

En este rally se plantean diversos retos, que los estudiantes tendrán que resolver en el transcurso de 36 horas aproximadamente. Además de los estudiantes, participan como mentores, docentes y otros profesionales que conducen el proceso de definición y opertivización de las ideas de los estudiantes. Los retos son variados y son del conocimiento del estudiantado que participa, hasta el momento que inicia el evento. No obstante, la sistematización de esta experiencia queda a nivel de informes, por lo que en este documento, se busca mostrar el efecto del 
Rally en el desarrollo de competencias genéricas y profesionales de los que participan en él.

En la Facultad Regional Multidisciplinaria de Chontales de la UNAN-Managua, en el año 2020, participaron 50 estudiantes, 8 tutores y 2 asesores. Uno de los trabajos ahí creados, recibió un premio nacional. No obstante, más allá de la competitividad, el significado de este concurso recae en el desarrollo académico y profesional de los participantes.

\section{Palabras Claves}

Competencias genéricas, competencias profesionales, rally latinoamericano, innovación

\section{Summary}

In this article, which arises from the systematization of an extension experience, it is tackled the main aspects of the Latin American innovation rally and its impact on the development of generic and professional competences in the students who participate in this event. The Latin American innovation rally takes place every year at the UNANManagua Faculties, as well as at other Latin American universities. The fundamental objective of the rally is the development of competences for innovation in students who are training in universities. This rally poses various challenges that students will have to solve over the course of approximately 36 hours. In addition to the students, professors and others proffesionals work as guide who lead the process of definition and operationalization of the students' ideas. The challenges are varied and are known to the participating students, until the event begins. However, the systematization of this experience remains at the reporting level, so this document seeks to show the effect of this Rally on the development of generic and professional competences in those who participate in it.

At the Facultad Regional Multidisciplinaria de Chontales, UNAN-Managua, in 2020, participated 50 students, 8 tutors and 2 advisers. One of the works created got a national award. However, beyond competitiveness, the significance of this event lies in the development of academic and professional skills in the participants.

\section{Keywords}

Generic competences, professional competences, latin american rally, innovation

\section{Introducción}

\section{Múltiples retos de las universidades en la actualidad}

Las universidades en estos momentos se encuentran en el epicentro de profundas reflexiones, donde, desde diversas perspectivas y con los más variados intereses, buscan repensar y replantear su papel en la construcción de conocimiento, que sirva de motor para el desarrollo de los países que tanto lo necesitan. Partiendo de esta premisa, el desarrollo de las universidades ha de coincidir con el conocimiento generado, y que este conocimiento sirva de fundamento para el planteamiento de soluciones innovadoras a los diferentes problemas que afectan a las sociedades (Marcano G, Rojas H, \& Masello, 2014).

La universidad está llamada a convertirse en una institución que ofrezca respuestas a las complejidades del mundo moderno, con aportes significativos para el desarrollo de los países en términos de superación de las desigualdades, pobreza, cambio climático y exclusión; junto con el aprovechamiento racional de los recursos que garantizan la supervivencia humana en armonía con el medio ambiente. Es aquí donde se ha de exigir que los perfiles de egreso apunten a formar profesionales competentes para identificar problemas y utilizar procesos creativos para el diseño y desarrollo de propuestas de solución a las problemáticas que se plantean en los entornos laborales, sociales y que éstas puedan ser resueltas en equipos multidisciplinares.

Las universidades como entes reguladores, deben aprovechar todas aquellas acciones extracurriculares surgidas en diversos contextos que buscan el fortalecimiento del currículum, en cuanto al desarrollo de competencias de corte académico y profesional. En este sentido, el Rally Latinoamericano de Innovación, es una actividad extra curricular en la que se insertan los estudiantes, para fortalecer sus conocimientos, además de ponerse en contacto con una metodología que permite la consecución de valores agregados como: la innovación y el emprendimiento.

Hay diversas reflexiones acerca de la formación profesional basada en competencias, entre ellas se 
destaca la de Gardner que refiere "las competencias no son innatas y las personas a partir de las exigencias y estímulos del entorno pueden desarrollar capacidades específicas" (1995, p.20) .Es decir, que es importante la realización de eventos con las características del Rally Latinoamericano de Innovación, que promuevan una cultura de innovación abierta con propuestas de solución a problemas reales, a su vez aportando ciertos estímulos para que el estudiante desarrolle su capacidad creativa y heurística.

Particularmente, en el Rally se potencia el trabajo en equipo conformados por protagonistas de diferentes áreas del saber, esto, complementa la educación formal a través de experiencias creativas e innovadoras que se requieren para la generación de emprendimientos y que, además, apuesta por la búsqueda de competencias genéricas como la comunicación por diversos medios, la negociación de ideas, la toma de decisiones y la comprensión. En otras palabras, la innovación y el emprendimiento requiere sobre todo voluntad y disposición de elevar el pensamiento humano al máximo para el desarrollo de ideas pertinentes y útiles.

En consonancia con lo anterior, el desarrollo de capacidades, como la del trabajo en equipo, es una competencia indispensable para el desempeño de manera efectiva de trabajo diversos. Por último, teniendo en consideración el formato de presentación de la resolución de los retos, en videos y formularios, para plantear las alternativas que dan salida a la idea del desafío, se fomenta el desarrollo de competencias para la comunicación precisa y efectiva, utilizando los recursos tecnológicos que la contemporaneidad les brinda. El impacto de la innovación y el emprendimiento sobre los negocios avanzan de una manera rápida y tiene un alcance tan extensivo, que obliga a actuar para lograr los resultados que un país, como Nicaragua, busca para la continuidad de su desarrollo socioeconómico.

\section{Papel de la Universidad en la formación de competencias para la innovación}

Se manifiestan momentos de cambios en todos los órdenes: social, cultural, tecnológico, y epistémico; cambios que cuestionan las formas tradicionales de realizar ciencia. Las universidades, siendo los espacios innatos donde se genera la ciencia y la tecnología, con la llegada de un mundo de conocimientos plurales, transitorios y sin fronteras, el debate se centra en la pertinencia de sus prácticas y en los productos del saber para responder a las necesidades del contexto laboral, científico y social. En este marco, unos declaran la agonía de la universidad, otros menos fatalistas, siguen viéndola como una corporación que está agotada en su principios, naturaleza y roles, pero que tiene posibilidades de recuperación al ser capaz de transformarse y ponerse al día para recuperar la pertinencia, intrepidez y significado que tiene para la sociedad.

La universidad está obligada a transformar su forma de enseñanza para dar respuestas a las complejidades del mundo actual, con aportes significativos para el desarrollo de los países en términos de superación de las desigualdades, mejora del medio ambiente, reducción de la pobreza y mitigación de la exclusión social; con acciones que garanticen la continuidad racionalmente útil de la humanidad. Acompañando esas transformaciones en los procesos de enseñanza, ha de estar la extensión universitaria, dado que es uno de los pilares sobre los que se sustenta la universidad contemporánea.

La universidad, como institución que aprende, ha de insertarse en todas las acciones extensionistas en pro del fortalecimiento de sus capacidades .En ese sentido, la UNAN-Managua, ha aprovechado con todas sus Facultades e Institutos, la oportunidad que genera el Rally Latinoamericano de Innovación, para poner en contacto al estudiante con realidades globales y nacionales, que le permita la potenciación de su conciencia social, a través del análisis de problemáticas locales y de otros contextos para el desarrollo de las competencias académicas y laborales necesarias.

\section{Material y Métodos}

Para valorar la percepción de los protagonistas en el Rally latinoamericano de innovación 2020 y el impacto que éste tuvo, en el fortalecimiento de sus competencias, se aplicó una encuesta que consta de tres apartados, uno de ellos, está orientado a la recolección de información sobre la opinión de los estudiantes que participaron en este evento, en lo que concierne al desarrollo de competencias genéricas y profesionales, que es el objeto de este artículo. La encuesta se aplicó a estudiantes, utilizando Google Form, luego los resultados se exportaron a una base de datos de SPSS 18, para la realización del análisis, utilizando elementos estadísticos de corte descriptivo. 
En la evaluación, se les pidió a los estudiantes expresar su grado de acuerdo con un conjunto de ítems, asignando una puntuación del uno al cinco, que estadísticamente se corresponde con quintiles, donde a mayor puntuación, mayor es el grado de acuerdo con cada afirmación expresada en el cuestionario.

Además, para medir la fiabilidad interna del instrumento, se aplicó alfa de Crombach y se obtuvo un resultado de 0.981 , con $\mathrm{N}=41$ elementos muestrales de un total de 50 elementos que tiene la población de estudio. Este valor obtenido, muestra un alto nivel de consistencia interna del instrumento aplicado. Como se está sistematizando una experiencia y este artículo, es el resultado de dicha actividad, el cuestionario se sometió al juicio de algunos compañeros con experiencia probada en el diseño de instrumentos de recolección de datos, para que se hicieran las observaciones pertinentes, es decir no fue necesario el criterio de expertos en la materia.

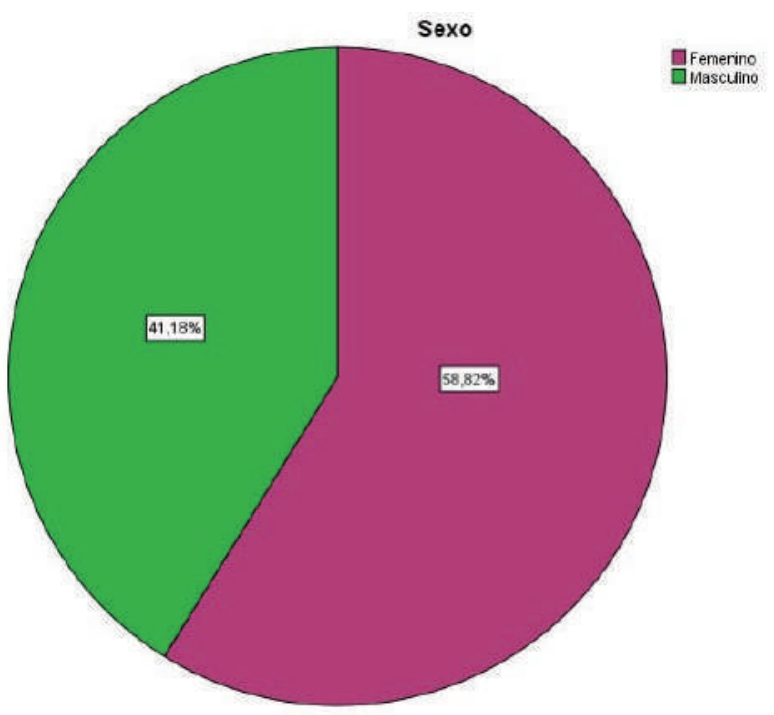

Gráfica 1. Disposición por sexo de los que participaron en el estudio. Fuente: Análisis descriptivo en SPSS

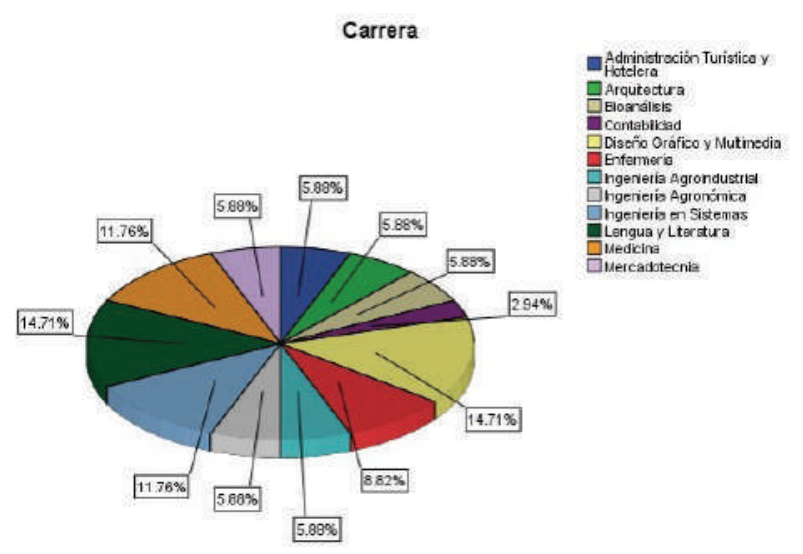

Gráfica 2. Disposición por carreras de los que participaron en el estudio Fuente: Análisis descriptivo en SPSS

Con la intención de presentar los resultados de forma compacta y concisa, se presenta una tabla (tabla $n^{\circ} 1$ ) en la cual, los porcentajes expresan la suma del cuarto y quinto quintil, que en términos porcentuales significa estar en un $80 \%$ de acuerdo con cada afirmación o totalmente de acuerdo equivale al $100 \%$ o sea el quinto quintil. En la tabla se muestran los porcentajes de opinión favorable de los estudiantes que participaron en el Rally, asi como los aspectos en el equipo de trabajo, consensuados como relevantes para ser sometidos a escrutinio y que, al medirse, recibieron puntuaciones de aceptación bastante altas, lo que muestra el impacto que tiene para los estudiantes y su proceso de formación integral, el haber participado en el Rally. 


\section{Criterios a medir}

1. Los retos planteados en el Rally, potencian el desarrollo de mi
creatividad.
$\begin{aligned} & \text { 2. Las áreas del conocimiento que se desarrollan fortalecen mi desempeño } \\ & \text { como futuro profesional }\end{aligned}$

3. Este concurso me permitió detectar oportunidades para emprender acciones de innovación.

4. El concurso ha desarrollado en mí, las capacidades de trabajo en equipo

5. Este concurso fortalece mi aprendizaje continuo.

6. El Rally despierta en mí, el espíritu emprendedor y mí necesidad de descubrimiento.

7. Este evento permitió autoevaluarme e identificar mis fortalezas y debilidades.

8. El Rally permitió descubrir mi capacidad de negociar ideas.

9. El Rally me permitió explorar oportunidades profesionales en el presente y para el futuro

10. El Rally, permitió desarrollar mis capacidades para enfrentar retos y trabajar por objetivos comunes.

11. El Rally permitió visionar mis capacidades de liderazgo.

12. El Rally me permitió crear y fortalecer relaciones de confianza y cooperación.

13. El Rally me permitió desarrollar la capacidad de trabajo en escenarios de incertidumbre

14. El Rally me permitió reconocer que el aprendizaje es continuo y que está en permanente evolución.

15. El Rally me permitió fortalecer el aprendizaje autónomo y la cooperación con el equipo de trabajo.

16. El Rally me permitió fortalecer el aprendizaje basado en el trabajo cooperativo.

17. El Rally me ha hecho ver que, en la innovación, son más importantes las ideas que los recursos.
91.2

Porcentaje de acuerdo

94.1

91.1

94.1

91.2

91.2

94.1

88.2

94.1

97.1

97.1

91.1

91.2

\section{1}

91.1

91.1

Tabla 1: Porcentajes de opinión favorable 
El desarrollo de competencias generales de corte comunicativo, es un asunto de mucho interés en la formación de profesionales del presente y para el futuro, no obstante, es importante entender qué es una competencia comunicativa. Por lo tanto, se abordaran algunos aspectos relevantes en los procesos de formación académica y profesional, relacionados con las competencias.

La competencia comunicativa, es una capacidad que comprende no sólo la habilidad lingüística, gramatical, de producir frases bien construidas y de saber interpretar y emitir juicios sobre frases producidas por el hablante al oyente o por otros, sino que, necesariamente, constará, por un lado, de una serie de habilidades extralingüísticas interrelacionadas, sociales y semióticas, y por el otro, de una habilidad lingüística polifacética y multiforme. (Navarro y Ortiz, 2010)

"La competencia comunicativa comprende las aptitudes y los conocimientos que un individuo debe tener para poder utilizar sistemas lingüísticos y translingüísticos, que están a su disposición para comunicarse como miembro de una comunidad sociocultural dada" (Navarro y Ortiz, 2010). Es decir que la competencia comunicativa exige comunicarse efectivamente, expresarse con autonomía y relacionarse con los demás. Es un acto de comprender, interpretar, analizar y producir diferentes tipos de textos según las necesidades de acción y comunicación.

La gráfica No. 3 que se presenta a continuación muestra un puntaje alto que expresa el acuerdo de los estudiantes que estaban en el Rally 2020 de la Facultad Regional Multidisciplinaria de Chontales, en cuanto al desarrollo de las competencias comunicativas, tanto de manera escrita, oral y virtual. Cabe señalar la importancia de la comunicación virtual en la actualidad, por su caracter determinante en el desarrollo de profesionales exitosos inmersos en un mundo propenso al uso de medios informáticos y del internet, para el teletrabajo, la comunicación de ideas, las inversiones, las investigaciones e innovaciones, los emprendimientos, la publicidad, etc.

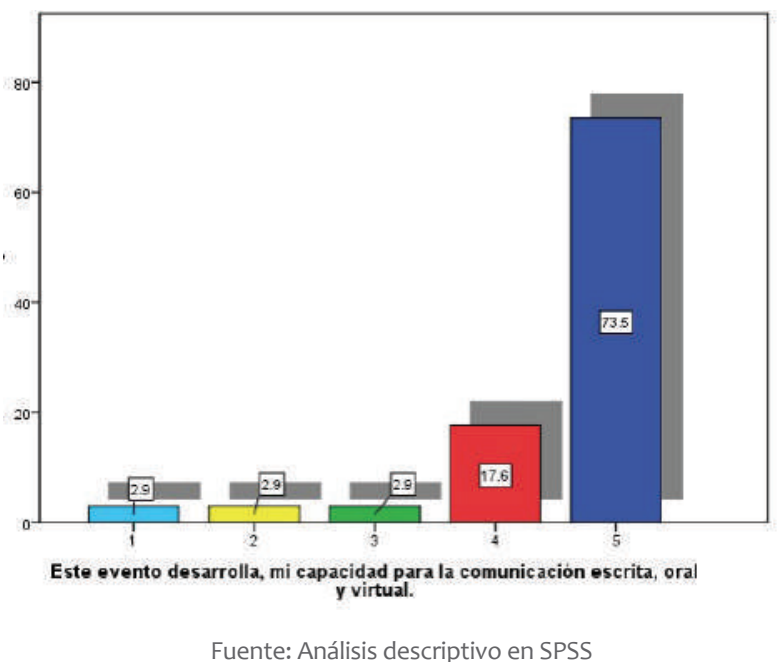

Al sumar los datos de la barra No. 4, con los de la barra No. 5, (cuarto y quinto quintil), se obtiene un $91.1 \%$, reflejando que los estudiantes que participaron en el Rally, 2020, perciben que éste les permitió el desarrollo de sus competencias de comunicación oral, escrita y por medios informáticos.

Uno de los aspectos más complicado en toda actividad humana, es la toma de decisiones. Las decisiones están supeditadas a una actitud inherente del ser humano: tratar de salir bien librado de las dificultades y quedar bien con los demás. En el campo académico, como en el laboral, no siempre habrán opiniones que tengan un grado de aceptacion en la comunidad. Y si a esto se le agrega, que puede ser desfavorable para los intereses personales, las cosas se complican más. El riesgo y la incertidumbre suelen estar presentes en todo acto decisivo, desde la asignación de recursos económicos, hasta la elaboración de programas de prevención de una política de salud pública; en la siembra de algodón, como en la promoción de campañas publicitarias para vender prendas confeccionadas con su fibra.

Las personas reaccionan de forma diferente ante una misma situación que involucra riesgos, dependiendo de si dicha situación es formulada en términos de ganar, de dejar de ganar o de perder. Algunos, ante un vaso por la mitad, harán un análisis muy simplista y dirán que está "medio vacío". Otros, con igual simplismo, argumentarán que, en realidad, el vaso está "medio lleno". Pero, seguramente no muchos serán lo suficientemente objetivos para darse cuenta que, tal vez, el vaso podría haber tenido la mitad del tamaño que tiene (Pilar, 2011). 
Estan compleja la toma de decisiones, que tiene ribetes de ciencia que se sustentan en métodos estadísticos. No obstante, cuando se adolece de los conocimientos científicos o se tienen esos conocimientos, pero por lo apremiante de la situación, no hay tiempo para hacer pruebas estadísticas, la mejor manera de tomar una decisión es sobre la base del consenso, si se está trabajando en equipo. En el Rally, los jóvenes toman decisiones trascendentes, sobre la base del diálogo y el consenso. Este es un valor agregado, que los jóvenes que participan en este evento, obtienen. Auscultamos la opinión de los jóvenes objeto de estudio, sobre si el Rally, les permitió aprender a tomar decisiones con información parcial y como se observa en la gráfica No. 4, donde se valora con un $97 \%$, esa afirmación, tomando en cuenta, lógicamente, los datos de cuarto y quinto quintil.

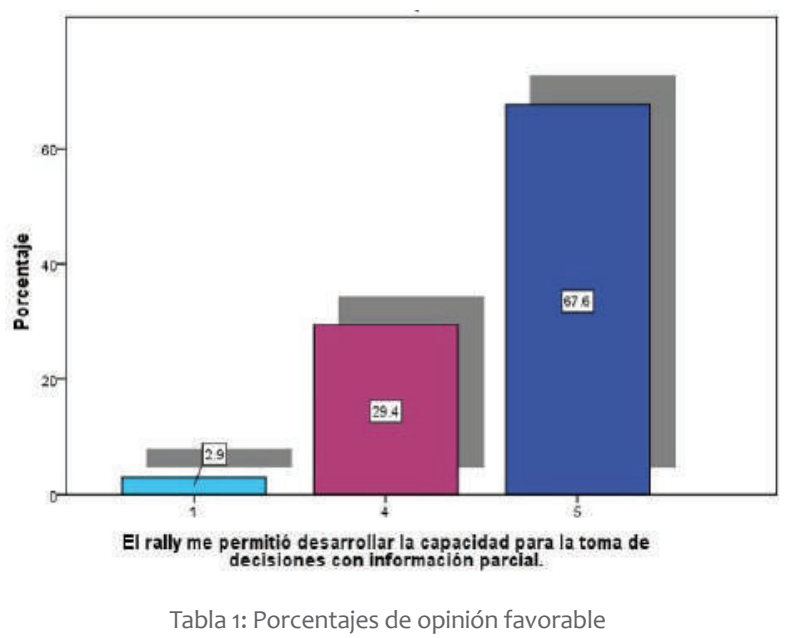

Sobre el mismo guion de la toma de decisiones, los estudiantes valoran positivamente, que el Rally les permitió valorar las bondades y riesgos inherentes a la toma de decisiones. Como se observa en la gráfica No. 5, la percepción favorable sobre esta afirmación supera el $90 \%$, tomando en cuenta, datos del cuarto y quinto quintil.

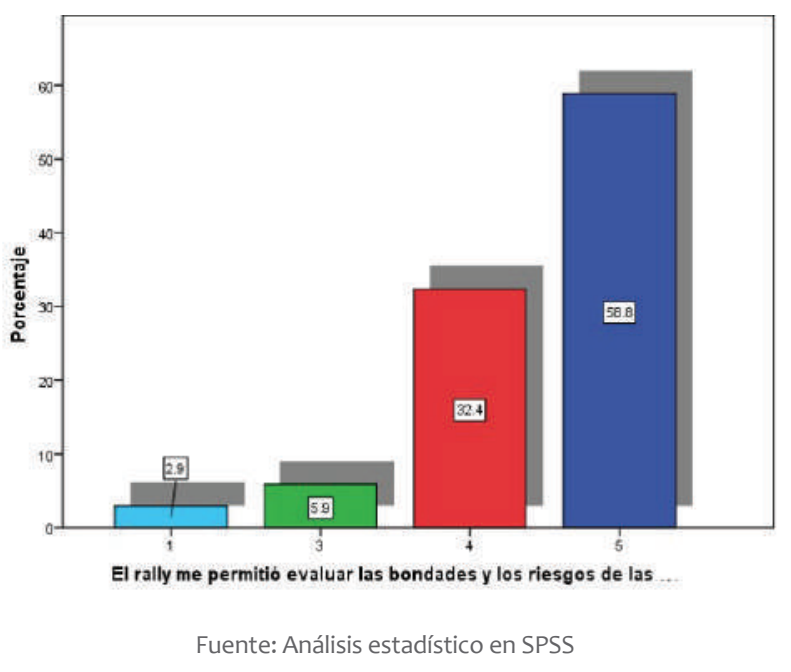

\section{Conclusiones}

Se concluye que el Rally, como actividad inmersa dentro de la extensión universitaria y según opinión de estudiantes de la FAREM-Chontales, que participaron en el Rally Latinoamericano de Innovación 2020, potencia la consecución de las siguientes capacidades y competencias:

- Los retos planteados en el Rally, potencian el desarrollo de la creatividad.

- Las áreas del conocimiento que se desarrollan fortalecen el desempeño como futuro profesional

- Este concurso permitió detectar oportunidades para emprender acciones de innovación.

- El concurso ha desarrollado las capacidades de trabajo en equipo.

- $\quad$ Este concurso fortalece el aprendizaje continuo.

- El rally despierta el espíritu de emprendedor y la necesidad de descubrimiento.

- Este evento desarrolla, la capacidad para la comunicación escrita, oral y virtual.

- Este evento permitió la autoevaluación e identificar las fortalezas y debilidades.

- El rally permitió descubrir la capacidad de negociar ideas. 
- El rally permitió explorar oportunidades profesionales en el presente y para el futuro

- El rally permitió desarrollar las capacidades para enfrentar retos y trabajar por objetivos comunes.

- Desarrolla capacidades de liderazgo.

- Permite el desarrollo de relaciones de confianza y cooperación.

- Desarrolla la capacidad para la toma de decisiones con información parcial.

- Desarrolla la capacidad de trabajo en escenarios de incertidumbre

- Permite comprender que el aprendizaje está en permanente evolución.

- Fortalecer el aprendizaje autónomo y la cooperación con el equipo de trabajo.

- Permite, al estudiante, evaluar las bondades y los riesgos de la toma de decisiones.

- Permite fortalecer el aprendizaje basado en el trabajo cooperativo.

- Ayuda a comprender que, en la innovación, son más importantes las ideas que los recursos.

\section{Referencias bibliográficas}

- Gardner, H. (1995). Inteligencia múltiple: La teoría en la práctica. Barcelona: Paidós. Recuperado el 07 de 10 de 2020 sitio web de donde se extrajo la cita

- Marcano G, D., Rojas H, L., \& Masello, S. (12 de noviembre de 2014)

- Navarro, L., \& Ortiz, A. (2010). ¿Cómo estimular el desarrollo de competencias comunicativas? Barranquilla: Educosta. $\mathrm{n}^{\circ}$ de página

- Organización de Estados Iberoamericanos, ISBN: 978-84-7666-210-6. Recuperado el 07 de 10 de 2020, de Organizacin de Estados Iberoamericanos: https://www.oei.es/acercade/que-es-la-oei

- Pilar, J. V (2011) Herramientas para la Gestión y la Toma de Decisiones. Buenos Aires: HANNE

- Vera Carrasco, O. (2015). La formación médica basada en competencias.

revistaboliviana.org.bo, 64. Recuperado el 13 de 10 de 2020 sitio web de donde se extrajo la cita 